\title{
Application Effect of Whole-Process Seamless Nursing Model Based on Smart Healthcare Mode in Perioperative Period of Patients Undergoing Hematoma Removal
}

\author{
Chang Liu, ${ }^{1}$ Rui Liu, ${ }^{2}$ Zhaohua Li, ${ }^{1}$ Meiling Tang, ${ }^{1}$ and Fenghua Wang $\mathbb{D D}^{3}$ \\ ${ }^{1}$ Operation Room, the First Affiliated Hospital of Harbin Medical University, Harbin 150001, Heilongjiang, China \\ ${ }^{2}$ Library Room, Harbin Children's Hospital, Harbin 150000, Heilongiang, China \\ ${ }^{3}$ Department of Medical Section, the First Affiliated Hospital of Harbin Medical University, Harbin 150001, Heilongjiang, China
}

Correspondence should be addressed to Fenghua Wang; wangfenghua@54dr.net.cn

Received 4 January 2022; Revised 17 January 2022; Accepted 26 January 2022; Published 23 February 2022

Academic Editor: Bhagyaveni M.A

Copyright (c) 2022 Chang Liu et al. This is an open access article distributed under the Creative Commons Attribution License, which permits unrestricted use, distribution, and reproduction in any medium, provided the original work is properly cited.

Objective. To explore the application effect of a whole-process seamless nursing model based on the smart healthcare mode in the perioperative period of patients undergoing hematoma removal. Methods. In this retrospective study, 50 patients with hematoma removal admitted to our hospital from August 2018 to August 2019 were included as the control group (CG), while 50 patients with hematoma removal admitted to our hospital from September 2019 to September 2020 were included as the experimental group (EG). During the period of hematoma removal, CG received routine perioperative nursing, while EG received the wholeprocess seamless nursing model based on the smart healthcare mode. The perioperative indexes, hemodynamic indexes, and the incidence of postoperative complications were compared between the two groups, and the incidence of nursing staff's work omissions in different periods was analyzed. Results. Notable differences were observed in surgical time, intraoperative blood loss, hematoma clearance rates, length of ICU stay, hospitalization time, removal time of ventricular drainage tube, and cerebral edema volume at 1 week after surgery between EG and CG $(P<0.05)$. Compared with CG, EG achieved obviously better hemodynamic indexes $(P<0.001)$ and a lower incidence of bedsore, muscle atrophy, and eating/swallowing disorders $(P<0.05)$. During the implementation of smart healthcare, the incidence of nursing staff's work omissions was remarkably reduced $(P<0.05)$. Conclusion. Under the smart healthcare, the incidence of nursing staff's work omissions is lower, and the effect of the wholeprocess seamless nursing is better, which can optimize the perioperative indexes of patients, stabilize the postoperative hemodynamics, and reduce the incidence of complications. Therefore, the whole-process seamless nursing model based on the smart healthcare mode has promotion value in clinic.

\section{Introduction}

Intracranial hematoma, the most common secondary lesion of craniocerebral injury, is often caused by abnormal accumulation of intracranial hemorrhage in the cranial cavity. The disease will cause increased intracranial pressure and brain tissue damage in patients, subsequently triggering a series of clinical symptoms such as disturbance of consciousness and memory decline. Without timely intervention, patients may suffer from complications such as cerebral ischemia and irreversible neurological dysfunction, which seriously endanger their life and health $[1,2]$. At present, its clinical treatments mainly include conservative treatment and surgical treatment, in which only surgical treatment can eliminate hematoma. Therefore, patients meeting the surgical indications without surgical contraindications should receive surgical treatment as soon as possible. In particular, hematoma removal should be performed in patients with severe craniocerebral injury to reduce the risk of death $[3,4]$.

Though hematoma removal can quickly remove the hematoma, alleviate the occupying effect, and reduce the pressure of hematoma on normal brain tissues, it puts forward higher requirements for nursing, and its combination with high-quality perioperative nursing can minimize 
the potential tissue damage and improve surgical outcomes. Camicia Michelle et al. have reported that seamless nursing is able to enhance the continuity of perioperative nursing and reduce patients' psychological pressure on surgery [5]. Some studies have applied it in hematoma removal and found that this nursing model reduces the incidence of postoperative complications such as postoperative pulmonary infection and muscular atrophy, improves the nursing satisfaction of patients, and reduces nurse-patient disputes $[6,7]$. In recent years, with the further promotion of the smart healthcare, many hospitals have developed an integrated platform of nursing information to share online medical resources and effectively solve the problem of difficult integration of nursing information among various systems. The data sharing and objectivity of smart healthcare bring more possibilities to the seamless nursing because the online platform can optimize the operation process, improve the nursing efficiency, and effectively reduce the possibility of nursing omissions $[8,9]$. On the basis of routine nursing assisted by smart healthcare platform, nursing staff only need to adopt the evidence-based nursing to gradually improve the seamless nursing model and deepen the nursing details, thereby comprehensively enhancing the service quality. At present, there is no research on smart healthcare platform as the basis of seamless nursing. Based on this, this paper combined the two in patients with hematoma removal to further improve the perioperative nursing effect.

\section{Materials and Methods}

2.1. Study Design. This retrospective study was conducted in our hospital from August 2018 to September 2020 to explore the application effect of a whole-process seamless nursing model based on the smart healthcare mode in the perioperative period of patients undergoing hematoma removal.

\subsection{Inclusion Criteria and Exclusion Criteria. Inclusion} criteria: (1) patients were confirmed with intracranial hematoma by head CT or MRI and underwent hematoma removal [10]; (2) patients are aged no less than 18 years old; (3) the time from onset to treatment of the patients was within 24 hours; and (4) patients were treated in our hospital throughout the whole process and had complete clinical data.

Exclusion criteria: (1) patients had severe organic diseases; (2) patients had mental illness or other factors affecting communication; (3) patients did not pass the drug sensitivity test; (4) patients had seriously abnormal coagulation function or took anticoagulants and antiplatelet drugs within one week after the onset; and (5) patients were in pregnancy or lactation.

2.3. General Data of Patients. Fifty patients with hematoma removal admitted to our hospital from August 2018 to August 2019 were included as the control group (CG), while 50 patients with hematoma removal admitted to our hospital from September 2019 to September 2020 were included as the experimental group (EG). No notable differences in general data were found between the two groups $(P>0.05)$, see Section 2.1 .

2.4. Ethical Consideration. This study was in line with the principles of Helsinki Declaration (2013) [11]. After the patients were recruited, the research group explained the study purpose, significance, content, and confidentiality to them and asked them to sign the informed consent.

2.5. Methods. The CG patients received routine perioperative nursing. Before surgery, the nursing staff performed preoperative education, carefully checked the patients' surgical information, and prepared the surgical instruments and drugs. During surgery, the staff closely monitored the patients' physical data such as heart rate, blood oxygen saturation, blood pressure, and body temperature and cooperated with the doctors to help patients maintain the appropriate position and protect their privacy. After surgery, the staff helped to wipe the blood on the patients, sent them to the wards, and did the handover. Then, the nursing staff in the wards closely observed the changes of patients' condition and signs.

The EG patients group received the whole-process seamless nursing model based on the smart healthcare mode, with the following measures based on the routine nursing in CG. (1) A seamless nursing group was established, including a senior and experienced nursing staff as the leader, a nursing staff with strong communication ability as the deputy leader, the head nurse of the department as the counselor, 20 nursing staff with professional training and specialist work experience over 3 years as the team members, and 2 nursing staff as the office staff. Through evidencebased nursing, team members consulted relevant guidelines and literature, consulted the experts, listened to related lectures, and cultivated awareness of smart healthcare. (2) The seamless nursing group and the R\&D personnel of the software development company jointly established the smart healthcare management platform, including four systems. One was the nursing workstation system, mainly including medical advice processing module, surgery appointment module, and medical instrument management module. The nursing staff accessed and operated the platform through the mobile client. The second one was laboratory and inspection configuration system. The office nursing staff generated project labels through computer operation after checking the medical orders of test or inspection in the medical advice processing module and arranged the patients for examination. The third was nursing information management system, including nursing electronic medical records, nursing quality control, head nurse scheduling, adverse event reporting, and nursing safety monitoring. The fourth was the electronic display board system. The nursing staff consulted patient information, shift information, patient vital sign monitoring data, catheter data, nursing assessment, relevant doctor's advice, nursing records, and test and examination results on the electronic display board. The above data were updated in real time, the nursing staff could modify the data on any interface, and the office nursing staff 
were responsible for checking. (3) The seamless nursing group logged into the smart healthcare management platform and reviewed and processed the patients' medical orders (nursing level, diet, laboratory test, and examination). Operated online by office nursing staff without manual entry and transcription, the platform automatically printed the orders into treatment sheets, infusion sheets, injection sheets, and appointment numbers. The perioperative nursing measures in the standardized process of seamless nursing for hematoma removal were directly generated by the smart healthcare system. (4) On the basis of routine nursing measures issued by the smart healthcare system, combined with the nursing risks in the operating room and the actual situation of patients with hematoma removal over the years, the group summarized the problems existing in the perioperative nursing, and then formulated a more detailed seamless nursing scheme, mainly including preoperative visits, intraoperative nursing and postoperative nursing. (5) Before surgery, the nursing staff performed health education to patients to alleviate their negative psychological state and help them to overcome fear. During surgery, the staff strictly controlled the temperature and humidity in the operating room and massaged the patients' limbs to promote blood circulation for those with long surgical time. After surgery, the staff did handover, helped patients wake up as soon as possible, wear clothes and cover the quilt, and fixed the drainage tube. After recovery, the staff visited the patients again to inform them of the possible postoperative complications and precautions and provided psychological comfort for them.

2.6. Observation Criteria. General data: after the patients were enrolled, the general data were collected, and the information files were established, including gender, age, height, weight, BMI, Glasgow Coma Scale (GCS) index [12], and hematoma volume.

Perioperative indexes: perioperative indexes included surgical time, intraoperative blood loss, hematoma clearance rate, length of ICU stay, hospitalization time, removal time of ventricular drainage tube, and cerebral edema volume at 1 week after surgery.

Hemodynamic indexes: electrocardiogram (TLC6000, Hebei Medical Products Administration certified No. 20102210047) was performed to monitor the patients' heart rate at 1 day before surgery and 1 day after surgery. The standard mercury sphygmomanometer (Jiangsu Yuwell Medical Equipment Co, Ltd.; Jiangsu Medical Products Administration certified no. 20152070945) was adopted to monitor the patients' blood pressure at 1 day before surgery and 1 day after surgery and to calculate the mean arterial pressure (MAP).

Incidence of postoperative complications: the complications included bedsore, muscle atrophy, pulmonary infection, eating/swallowing disorders, hypostatic pneumonia, acute ulcer hemorrhage of digestive tract, and joint stiffness. The number of patients with complications was recorded.

Incidence of work omissions: the 24 nursing staff's work omissions from August 2018 to August 2019 and from
September 2019 to September 2020 were recorded, including transcribe errors of infusion sheets, transcribe errors of doctor's advice, errors or omissions of infusion sheets, errors or omissions of laboratory labels, information errors of electronic display board, and nurse-patient disputes.

2.7. Statistical Treatment. The data in this study were processed by the SPSS20.0 software and graphed by GraphPad Prism 7 (GraphPad Software, San Diego, USA). This study included enumeration data and measurement data, tested by $\mathrm{X}^{2}$ and $t$-test. The differences were statistically significant at $P<0.05$.

\section{Results}

3.1. General Data. The EG had 30 males and 20 females, with an average age of $44.02 \pm 16.86$ years old, average height of $172.65 \pm 15.11 \mathrm{~cm}$, average body weight of $60.98 \pm 5.65 \mathrm{~kg}$, average BMI of $22.65 \pm 2.32 \mathrm{~kg} / \mathrm{m}^{2}$, average GCS score of $7.22 \pm 1.10$, and average hematoma volume of $50.22 \pm 10.26$ $\mathrm{mL}$. CG had 28 males and 22 females, with an average age of $43.96 \pm 17.42$ years old, average height of $172.78 \pm 15.23 \mathrm{~cm}$, average body weight of $61.18 \pm 5.60 \mathrm{~kg}$, average BMI of $22.41 \pm 2.30 \mathrm{~kg} / \mathrm{m}^{2}$, average GCS of $7.25 \pm 1.10$, and average hematoma volume of $50.12 \pm 10.28 \mathrm{ml}$. No notable differences in general data were found between the two groups $(P>0.05)$.

3.2. Perioperative Indexes. Notable differences in the perioperative indexes were observed between EG and CG $(P<0.05)$, see Table 1.

3.3. Hemodynamic Indexes. The postoperative hemodynamic indexes were notably better in EG than in CG $(P<0.001)$, as presented in Figure 1.

No notable differences in heart rates and MAP were observed between EG and CG at 1 day before surgery $(69.23 \pm 2.65$ vs. $69.50 \pm 2.44$ and $85.65 \pm 5.20$ vs. $87.00 \pm 5.23 ; P>0.05)$. The heart rate and MAP in EG were obviously lower than those in CG at 1 day after surgery $(72.11 \pm 2.65$ vs. $78.65 \pm 2.68$ and $89.68 \pm 5.65$ vs. $95.11 \pm 5.47 ; P<0.001)$.

3.4. Incidence of Postoperative Complications. The incidence of bedsore, muscular atrophy, and eating/swallowing disorders in EG was remarkably lower compared with CG $(P<0.05)$, see Table 2 .

3.5. Incidence of Work Omissions. During the implementation of smart healthcare, the incidence of nursing staff's work omissions was remarkably reduced $(P<0.05)$, as presented in Table 3.

\section{Discussion}

Intracranial hematoma is the abnormal blood aggregation caused by vascular rupture between brain or brain tissues and skull. The disease includes acute intracranial hematoma 
TABLE 1: Comparison of patients' perioperative indexes.

\begin{tabular}{|c|c|c|c|c|}
\hline Group & $\mathrm{EG}(n=50)$ & CG $(n=50)$ & $\mathrm{X}^{2} / t$ & $P$ \\
\hline Surgical time (min) & $78.65 \pm 10.22$ & $123.32 \pm 15.32$ & 17.152 & $<0.001$ \\
\hline Intraoperative blood loss $(\mathrm{mL})$ & $44.21 \pm 5.65$ & $154.98 \pm 13.65$ & 53.019 & $<0.001$ \\
\hline Hematoma clearance rate $(\%)$ & $96.0(48 / 50)$ & $84.0(42 / 50)$ & 4.000 & 0.046 \\
\hline Length of ICU stay $(\mathrm{d})$ & $2.67 \pm 0.54$ & $3.10 \pm 0.35$ & 4.725 & $<0.001$ \\
\hline Hospitalization time $(\mathrm{d})$ & $7.32 \pm 1.51$ & $9.24 \pm 1.23$ & 6.971 & $<0.001$ \\
\hline Removal time of ventricular drainage tube $(\mathrm{d})$ & $3.44 \pm 0.84$ & $4.96 \pm 0.65$ & 10.119 & $<0.001$ \\
\hline Cerebral edema volume at 1 week after surgery $(\mathrm{mL})$ & $24.12 \pm 2.55$ & $28.65 \pm 2.10$ & 9.697 & $<0.001$ \\
\hline
\end{tabular}

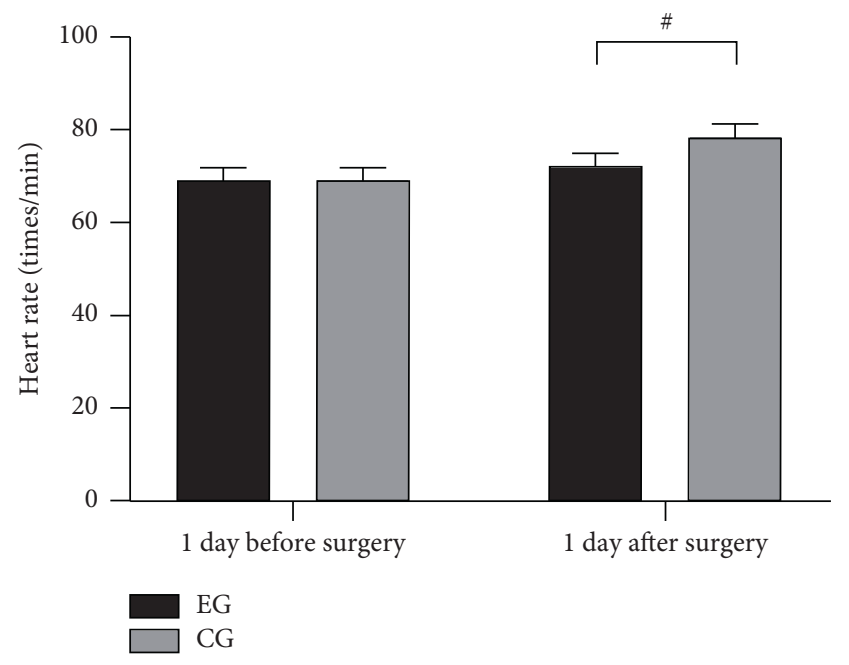

(a)

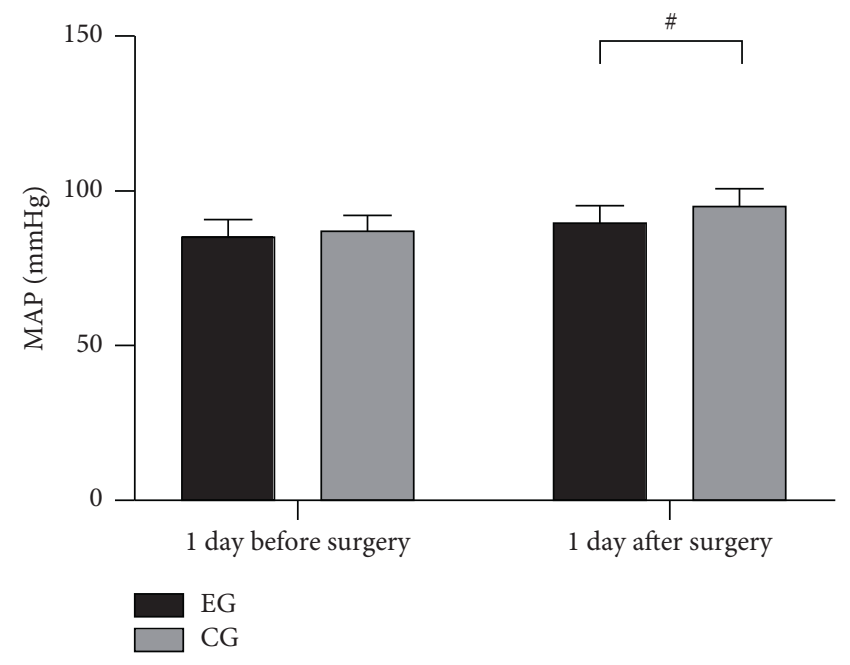

(b)

Figure 1: Comparison of patients' hemodynamic indexes $(-x \pm s)$. Note: the abscissa of Figures $1(\mathrm{a})$ and $1(\mathrm{~b})$ from left to right represented 1 day before and 1 day after surgery. The black area denotes EG, while the gray area denotes CG. \# indicates $P<0.001$.

TABLE 2: Comparison of the incidence of postoperative complications $(n(\%))$.

\begin{tabular}{|c|c|c|c|c|}
\hline Group & EG $(n=50)$ & CG $(n=50)$ & $\mathrm{X}^{2}$ & $P$ \\
\hline Bedsore & $0(0.0)$ & $4(8.0)$ & 4.167 & 0.041 \\
\hline Muscle atrophy & $0(0.0)$ & $4(8.0)$ & 4.167 & 0.041 \\
\hline Pulmonary infection & $1(2.0)$ & $3(6.0)$ & 1.042 & 0.307 \\
\hline Eating/swallowing disorders & $0(0.0)$ & $4(8.0)$ & 4.167 & 0.041 \\
\hline Hypostatic pneumonia & $0(0.0)$ & $3(6.0)$ & 3.093 & 0.079 \\
\hline Acute ulcer hemorrhage of digestive tract & $0(0.0)$ & $3(6.0)$ & 3.093 & 0.079 \\
\hline Joint stiffness & $1(2.0)$ & $5(10.0)$ & 2.837 & 0.092 \\
\hline
\end{tabular}

TABle 3: Comparison of the incidence of nursing staff's work omissions in different periods [n(\%)].

\begin{tabular}{|c|c|c|c|c|}
\hline Group & $\begin{array}{l}\text { September } 2019 \text { to September } 2020 \\
(n=24)\end{array}$ & $\begin{array}{l}\text { August } 2018 \text { to August } 2019 \\
\qquad(n=24)\end{array}$ & $\mathrm{X}^{2}$ & $P$ \\
\hline Transcribe errors of infusion sheets & $0(0.0)$ & $1(4.2)$ & 1.021 & 0.312 \\
\hline Transcribe errors of doctor's advice & $1(4.2)$ & $2(8.3)$ & 0.356 & 0.551 \\
\hline Errors or omissions of infusion sheets & $0(4.0)$ & $2(8.3)$ & 2.087 & 0.149 \\
\hline Errors or omissions of laboratory labels & $1(4.2)$ & $2(8.3)$ & 0.356 & 0.551 \\
\hline $\begin{array}{l}\text { Information errors of electronic display } \\
\text { board }\end{array}$ & $0(0.0)$ & $3(12.5)$ & 3.200 & 0.074 \\
\hline Nurse-patient disputes & $0(0.0)$ & $2(8.3)$ & 2.087 & 0.149 \\
\hline No omissions & $23(95.8)$ & $18(75.0)$ & 4.181 & 0.041 \\
\hline
\end{tabular}


and spontaneous intracranial hemorrhage, in which the former is caused by skull deformation and fractures after traumas and the latter is caused by diseases such as hypertension and cerebrovascular diseases $[13,14]$. The manifestations vary in the different types of hematoma, but most patients have varying degrees of consciousness disorder and a small number of patients are complicated with symptoms such as mental retardation and memory decline, which should be timely treated according to the different conditions of patients. For patients with less blood loss and mild craniocerebral injury, conservative treatment is generally adopted. For patients with supratentorial hemorrhage above $30 \mathrm{~mL}$, subtentorial hemorrhage above $10 \mathrm{~mL}$ or with cerebral hernia, hematoma removal should be performed immediately [15-17]. Hematoma removal is an important measure to reduce the changes of secondary diseases, with high requirements for nursing. Therefore, excellent perioperative nursing can effectively improve the surgical indicators of patients and boost the therapeutic effect [18]. At present, some scholars have applied seamless nursing in patients with hypertension undergoing craniotomy hematoma removal and achieved excellent results in reducing postoperative complications and improving the nurse-patient relationship. With the people-oriented nursing concept throughout the whole process, this nursing model aims to provide complete and continuous nursing services for patients. Additionally, the nursing staff need to constantly find problems and defects in the nursing process and then make modifications, thereby improving the nursing quality and eliminating the disadvantages of nursing services. The study of Xiao Feina and others has shown that seamless nursing improves the perioperative indexes of patients, with an average surgical time of $80.61 \pm 10.54 \mathrm{~min}$ [19]. This study also achieved the same results, indicating that seamless nursing has exact value in the perioperative nursing of patients undergoing hematoma removal. However, the seamless nursing model is rarely applied in practice, and its application is subject to various factors, among which the most critical factor lies in the cooperative work of nursing staff and the sharing of nursing information. Without highspeed and accurate intercommunication of medical resources, seamless nursing cannot play its practical role.

In recent years, the development of smart healthcare has provided more possibilities for the application of seamless nursing. Characterized by digitalization, automation, and intelligence, the smart healthcare management platform can standardize the nursing information system and simplify the nursing management process, especially avoiding the potential safety hazards caused by human factors in nursing work and shortening the handover time of nursing staff [20-22]. The application of smart healthcare management in seamless nursing can effectively improve the efficiency of collaborative work among nursing staff and increase the timeliness and scientificity of nursing work. With the support of this kind of information technology, nursing staff have fewer work omissions $[23,24]$. Therefore, the incidence of work omissions during the implementation of smart healthcare mode was significantly reduced $(P<0.05)$. Since the smart healthcare system has issued a routine process of seamless nursing, the workload of nursing staff is greatly reduced. The nursing staff can spend more time on the evidence-based nursing, learn from previous nursing, optimize seamless nursing measures, and provide patients with comprehensive and high-quality nursing [25]. Therefore, the patients in EG had more stable physical indicators after surgery and a lower incidence of bedsore, muscle atrophy and eating/swallowing disorders, suggesting that smart healthcare is a good basis for seamless nursing and can improve the application effect of this nursing model.

Hematoma removal is an important way to treat intracranial hematoma. Under the smart healthcare, the incidence of nursing staff's work omissions is lower, and the effect of the whole-process seamless nursing is better, which can optimize the perioperative indexes of patients, stabilize the postoperative hemodynamics, and reduce the incidence of complications. Therefore, the whole-process seamless nursing model based on the smart healthcare mode has promotion value in clinic.

\section{Data Availability}

Data to support the findings of this study are available on reasonable request from the corresponding author.

\section{Conflicts of Interest}

The authors have no conflicts of interest to declare.

\section{References}

[1] C. He, G. Yang, and M. Zhao, "A clinical study of high-dose urokinase for the treatment of the patients with hypertension induced ventricular hemorrhage," Progress in Brain Research, vol. 266, pp. 349-355, 2021.

[2] M. Gong, H. Zhang, Z. Shi, Q. Yuan, and X. Su, “Application of intraoperative ultrasound in neurosurgery for hypertensive intracerebral hemorrhage," Journal of Clinical Neuroscience, vol. 90, pp. 251-255, 2021.

[3] J. Su, Y. Xing, S. Liang, Q. Lin, and H. Liu, "Transcortical approach surgery versus external ventricular drainage in treating intraventricular hemorrhage," Medicine (Baltimore), vol. 100, Article ID e27443, 2021.

[4] O. Daniel Ayo, M. Elisha Didam, and M. Abu-Mahfouz Adnan, "Adaptability of assistive mobility devices and the role of the internet of medical things: comprehensive review," JMIR Rehabil Assist Technol, vol. 8, Article ID e29610, 2021.

[5] C. Michelle, L. Barbara, S. Debbie, L. Klassman, and S. Vaughn, "Nursing's role in successful stroke care transitions across the continuum: from acute care into the community," Stroke, vol. 52, 2021.

[6] D. Dharminder, U. Kumar, and P. Gupta, "A construction of a conformal Chebyshev chaotic map based authentication protocol for healthcare telemedicine services," Complex Intell Systems, vol. 7, pp. 1-12, 2021.

[7] X. Qian, S. Lan, and X. Zhang, "Effects of mild hypothermia therapy combined with minimally invasive debridement in patients with hypertensive intracranial hemorrhage: a randomized controlled study," Am J Transl Res, vol. 13, pp. 7997-8003, 2021.

[8] X. Lu, H. Junyi, and B. Shan, A. Gu, Determinants of the intention to use smart healthcare devices: a framework and 
public policy implications," J Healthc Eng, vol. 2021, Article ID 4345604, 7 pages, 2021.

[9] B. Gayathri Victoria, B. Paul, and C. Ruth, "Digital personal assistants are smart ways for assistive technology to aid the health and wellbeing of patients and carers," BMC Geriatrics, vol. 21, p. 643, 2021.

[10] D. El Majdoubi, E. Bakkali Hanan, S. Souad, and S. M. Chain, "A blockchain-based privacy-preserving smart healthcare framework," J Healthc Eng, vol. 2021, Article ID 4145512, 19 pages, 2021.

[11] World Medical Association, "World Medical Association Declaration of Helsinki: ethical principles for medical research involving human subjects," JAMA, vol. 310, no. 20, pp. 2191-2194, 2013.

[12] P. Jin, S. Deng, S. Prativa et al., "Neurokinin receptor 1 (NK1R) antagonist aprepitant enhances hematoma clearance by regulating microglial polarization via $\mathrm{PKC} / \mathrm{p} 38 \mathrm{MAPK} /$ $\mathrm{NF} \kappa \mathrm{B}$ pathway after experimental intracerebral hemorrhage in mice," Neurotherapeutics, vol. 18, 2021.

[13] W. Liu, L. Yin, C. Wang, L. Fulin, and N. Zhiyu, "Multitask healthcare management recommendation system leveraging knowledge graph," J Healthc Eng, vol. 2021, Article ID 1233483, 12 pages, 2021.

[14] P. Ivan Miguel, D. Hanna Vitaliyivna, V. M. Vanessa et al., "Mobile 5P-medicine approach for cardiovascular patients," Sensors, vol. 21, 2021.

[15] L. Jiang, F. Qian, W. Song, Y. Jiang, J. Pan, and G. Song, "Effect of nursing mode under the seamless connection between operating room and ward on severe traumatic brain injury patients in coma," Am J Transl Res, vol. 13, pp. 5087-5093, 2021.

[16] F. Ye, Y. Hua, F. Keep Richard, G. Xi, and H. J. L. Garton, "CD47 blocking antibody accelerates hematoma clearance and alleviates hydrocephalus after experimental intraventricular hemorrhage," Neurobiology of Disease, vol. 155, Article ID 105384, 2021.

[17] S. Mary Jo, M. McManus, A. Allen, J. Bethany, and P. Dennielle, "SmartPhrase: development of an electronic health record system to promote chemotherapy and immunotherapy safety," Clinical Journal of Oncology Nursing, vol. 25, pp. 85-88, 2021.

[18] C. F. Li, X. J. Lu, G. H. Wang et al., "Efficacy and safety of intraoperative ultrasound-assisted neuroendoscopy in the treatment of hypertensive intracerebral hemorrhage via transsylvian approach," Zhonghua Yixue Zazhi, vol. 101, pp. 620-623, 2021.

[19] F. Xiao, H. Peng, and Y. Li, "The preventive effect of seamless nursing care on pressure ulcer and related complications in elderly inpatients," Am J Transl Res, vol. 13, pp. 3515-3521, 2021.

[20] G. Sun, T. Fu, Z. Liu et al., "The rule of brain hematoma pressure gradient and its influence on hypertensive cerebral hemorrhage operation," Scientific Reports, vol. 11, p. 4599, 2021.

[21] E. Mun, J. Hong, S. Kwon, S. Ho Lee, W. Kim, and C. Lee, "Digital health care and arrhythmia: a case of WPW syndrome in South Korea, 2020," Ann Occup Environ Med, vol. 33, p. e8, 2021.

[22] K. Takeshi, N. Masato, S. Norihito, and O. Hiroki, "Effect of surgical arachnoid plasty on functional outcome in aneurysmal subarachnoid hemorrhage," World Neurosurg, vol. 147, pp. e373-e381, 2021.

[23] B. Theresa Sophie, K. Sven, N. Larissa, P. Rebacz, I. Kickbusch, and J. P. Ehlers, "Views on using social robots in professional caregiving: content analysis of a scenario method workshop," Journal of Medical Internet Research, vol. 23, Article ID e20046, 2021.

[24] J. Zhuang and Y. Peng, C. Gu, H. Chen, Z. Lin et al., Wogonin accelerates hematoma clearance and improves neurological outcome via the PPAR- $\gamma$ pathway after intracerebral hemorrhage," Transl Stroke Res, vol. 12, pp. 660-675, 2021.

[25] K. Byeong-Heon, J. S. Park, R. Mun-Ho, P. Chan, K. Y. Seop, and K. Jong-Dae, "Cloud-based software architecture for fully automated point-of-care molecular diagnostic device," Sensors, vol. 21, 2021. 